\title{
ANÁlise GRÁFICA E ANALÍTICA DA RETA DE EULER E TRÊS PONTOS NOTÁVEIS, EM TRIÂNGULOS NO ESPAÇO R²
}

\author{
P.C. SZENDRODI, J. ABRANTES, R.M. GRANADO, D. D. SOBRAL FILHA*
}

\begin{abstract}
Resumo
Este artigo faz análises gráfica e analítica, estabelecendo uma correspondência entre Álgebra e Geometria, tanto sobre a chamada Reta de Euler, quanto de relações proporcionais destes três Pontos Notáveis, em triângulos, no Espaço Bidimensional (X, Y), ou seja, Espaço $\mathrm{R}^{2}$. A análise gráfica, foi feita por desenhos executados com o programa AutoCAD®), versão 2015. Este artigo analisa as seguintes conclusões de Euler e outros renomados matemáticos, aplicadas aos triângulos: 1) Existe uma Reta que passa pelos seguintes Pontos Notáveis de triângulos escalenos e isósceles: Ortocentro $(\mathrm{O})$, Baricentro (G) e Circuncentro (C). Esta é a chamada Reta de Euler, que não se aplica a triângulos equiláteros. 2) Na Reta de Euler, o Baricentro (G) está localizado entre o Ortocentro (O) e o Circuncentro (C). 3) A distância entre o Baricentro $(\mathrm{G})$ e o Ortocentro (O) é o dobro da distância entre o Baricentro (G) e o Circuncentro (C). 4) Existe uma Circunferência com centro no encontro das Mediatrizes (o Circuncentro) e que passa pelos três vértices de um triângulo. Este artigo tem como objetivo ajudar a um melhor desenvolvimento das habilidades lógico-matemática e viso-espacial, tanto de estudantes da Licenciatura, quanto de professores de Matemática.
\end{abstract}

\section{Introdução}

Todo triângulo possui os chamados quatro Pontos Notáveis quais sejam: Ortocentro (O) como encontro das três alturas, Baricentro $(\mathrm{G})$ como encontro das três medianas; Circuncentro (C) como encontro das três mediatrizes e Incentro (I) como encontro das três bissetrizes. Leonhard Paul Euler (1707-1783) demonstrou que, em triângulos escalenos e isósceles quaisquer, existe uma Reta (chamada de Reta de Euler) que passa pelos seguintes três Pontos Notáveis: Ortocentro (O), Baricentro (G) e Circuncentro (C). Como em triângulos equiláteros estes três pontos coincidem (bem como o Incentro), fica óbvio que, nestes casos, não existe a chamada Reta de Euler, já que por um único ponto é possível passar-se uma infinidade de Retas. Este artigo faz análises Gráfica e Analítica, estabelecendo uma correspondência entre Álgebra e Geometria, que comprovam, tanto a Reta de Euler, quanto algumas relações proporcionais relacionadas aos três Pontos Notáveis, usando um triângulo escaleno, pertencente ao Espaço R2. A análise Gráfica foi obtida com desenhos geométricos, utilizando-se o programa AutoCAD®, versão 2015.

Palavras-chave: Geometria Plana; Pontos Notáveis nos Triângulos; Geometria Analítica; Reta de Euler.

Departamento de Representação Gráfica - IME/UERJ. pcs@ime.uerj.br, abrantes@ime.uerj.br, $\underline{\text { rmgra@ime.uerj.br e doraliceduque@ime.uerj.br }}$ 


\section{Objetivo e justificativa}

Este artigo objetiva mostrar, tanto por representação gráfica, via programa de computador, quanto por cálculos analíticos, estabelecendo uma correspondência entre Álgebra e Geometria, que existe uma Reta que passa pelos três Pontos Notáveis, bem como algumas relações proporcionais entre estes Pontos e elementos do triângulo. A principal justificativa para esta análise é um melhor desenvolvimento das habilidades lógico-matemática e viso-espacial, que em muito ajudam o ensino/aprendizado da Matemática, em especial da Geometria Analítica.

\section{Resumo da imagem geométrica e das análises/conclusões deste artigo}

As análises e conclusões deste artigo podem ser resumidas, segundo a Figura 1 a seguir, do triângulo $\mathrm{ABD}$, utilizado neste artigo.

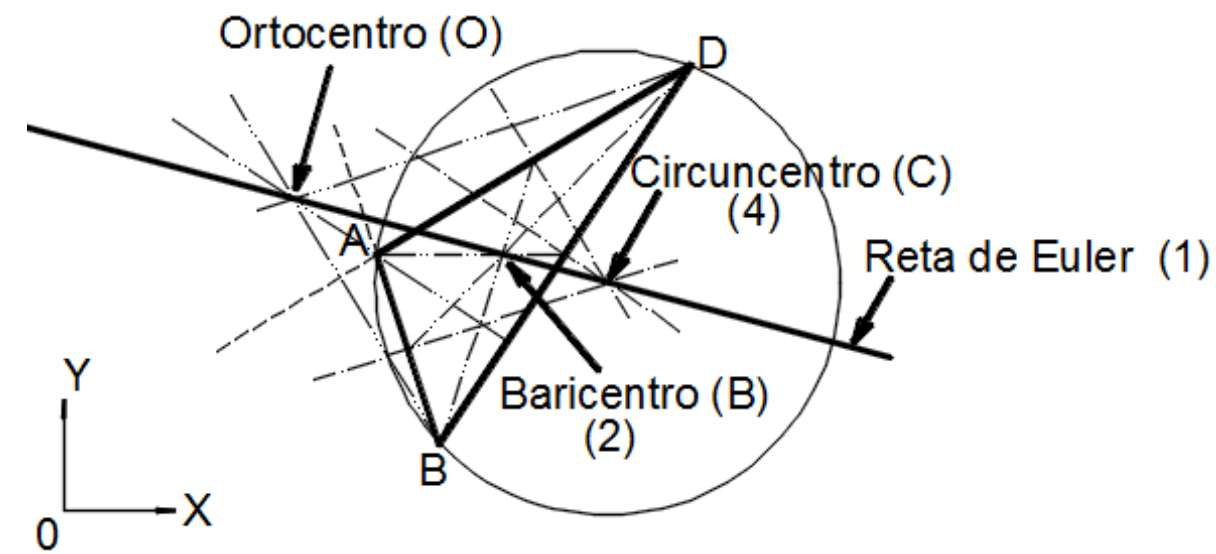

Figura 1 - Triângulo ABD.

Conclusão 1: existe uma Reta que passa pelos seguintes Pontos Notáveis de triângulos escalenos e isósceles: Ortocentro (O), Baricentro (G) e Circuncentro (C).

Conclusão 2: o Baricentro $(\mathrm{G})$ está localizado entre o Ortocentro $(\mathrm{O})$ e o Circuncentro (C).

Conclusão 3: a distância entre o Baricentro $(\mathrm{G})$ e o Ortocentro $(\mathrm{O})$ é o dobro da distância entre o Baricentro (G) e o Circuncentro, ou seja: $\mathrm{OG}=2(\mathrm{GC})$.

Conclusão 4: existe uma Circunferência com centro no encontro das Mediatrizes (Ponto C) e que passa pelos três vértices de um triângulo, ou seja: $\mathrm{CA}=\mathrm{CB}=\mathrm{CD}$.

\section{Características geométricas e análises gráficas do triângulo estudado}

Foi definido um triângulo escaleno de vértices $\mathrm{A}, \mathrm{B}, \mathrm{D}$, com as seguintes Coordenadas Cartesianas no Espaço Bidimensional ou R2: A $(+5 ;+4)$, B $(+6 ;+1)$ e D $(+10 ;+7)$ e representado na Figura 2 a seguir. 
P.C. Szendrodi, J. Abrantes, R.M. Granado, D. D. Sobral Filha
Análise Gráfica e Analítica da Reta de Euler e Três Pontos Notáveis, em Triângulos no Espaço $R^{2}$

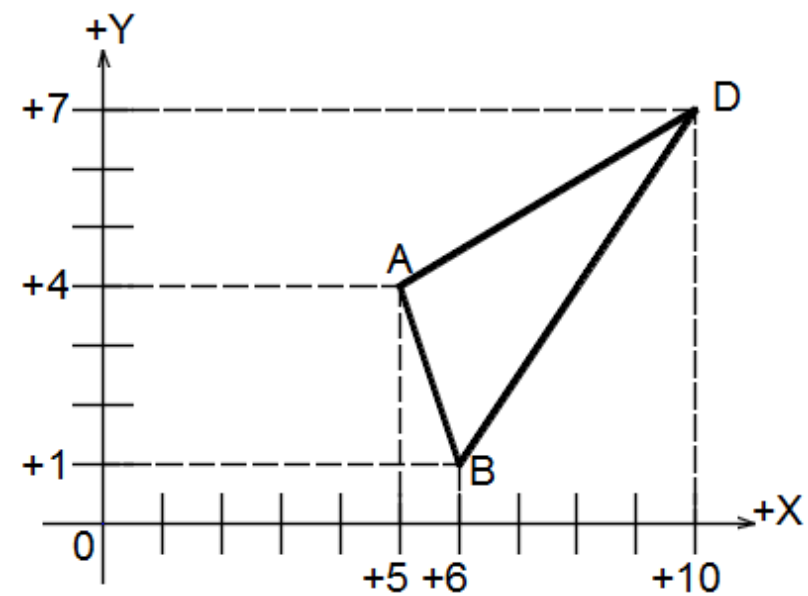

$\mathrm{AB}=3,162$

$\mathrm{AD}=5,831$

$\mathrm{BD}=7,211$

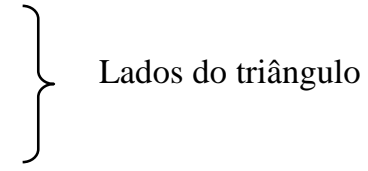

Imagem Geométrica do triângulo escaleno estudado
$A(+5 ;+4)$
B $(+6 ;+1)$
D $(+10 ;+7)$

Figura 2 - Triângulo escaleno de vértices A, B, D.

\subsection{Análise Gráfica do encontro das Alturas ou Ortocentro $(O)$}

Conforme pode ser observado na Figura 3 a seguir, em qualquer triângulo, o Ortocentro $(\mathrm{O})$ é o ponto do encontro das três alturas (CARVALHO, 2005, p.25). Altura é a distância entre um lado e o vértice oposto. $\mathrm{DE}=$ Altura em relação a $\mathrm{AB} ; \mathrm{BH}=$ Altura em relação a $\mathrm{AD}$ e $\mathrm{AF}=$ Altura em relação a $\mathrm{BD}$.
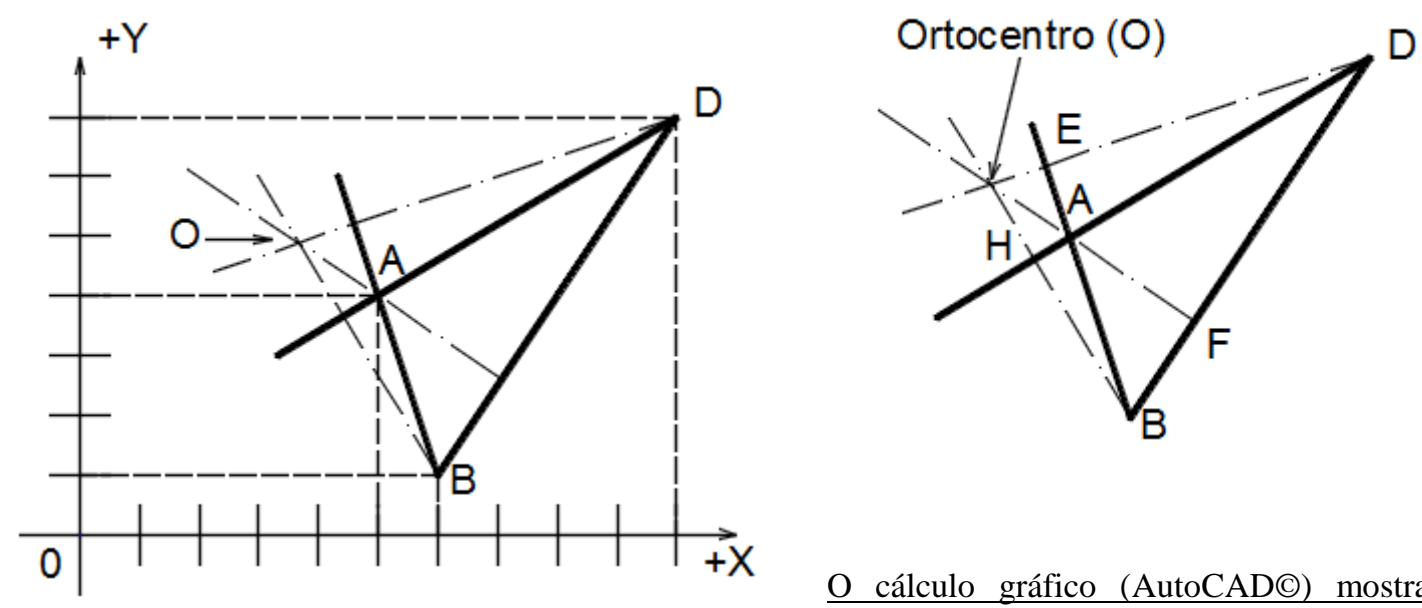

O cálculo gráfico (AutoCADC) mostra que as Coordenadas Cartesianas do Ortocentro são: $\underline{O}(+3,667 ;+4,89)$.

Figura 3 - Ortocentro $(\mathrm{O})$ do triângulo escaleno de vértices A, B, D.

\subsection{Análise Gráfica do encontro das Medianas ou Baricentro (G)}

Em qualquer triângulo, o Baricentro (G) é o ponto de encontro das três medianas (CARVALHO, 2005, p.25). $\mathrm{DI}=$ Mediana de $\mathrm{AB} ; \mathrm{BJ}=$ Mediana de $\mathrm{AD}$ e $\mathrm{AK}=$ Mediana de $\mathrm{BD}$. Mediana é a linha entre um vértice e o ponto médio do lado oposto. A Figura 4, a seguir, ilustra os detalhes acima apresentados. 


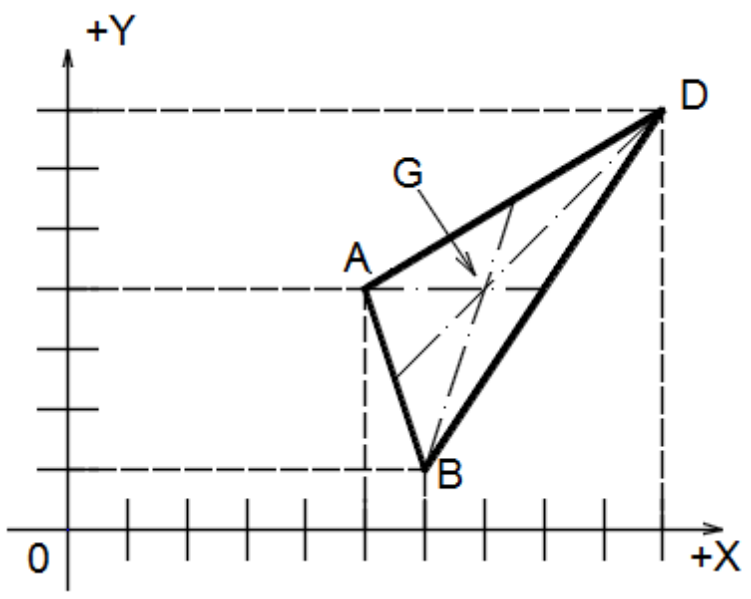

O AutoCAD mostra que as medianas são divididas em partes proporcionais, sendo uma, dois terços da outra, ou seja:

$\mathrm{GA}=2 / 3(\mathrm{AK})$.

$\mathrm{GB}=2 / 3(\mathrm{BJ})$.

$\mathrm{GD}=2 / 3(\mathrm{DI})$.

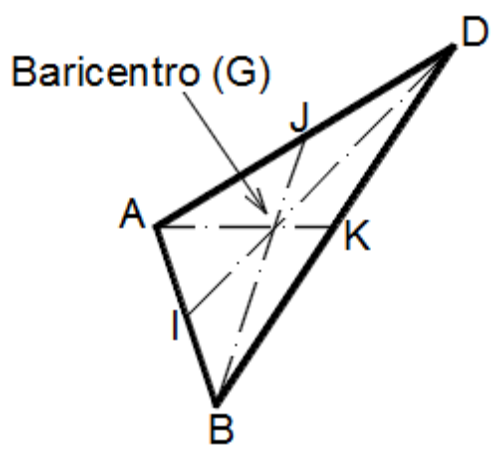

O cálculo gráfico (AutoCADC) mostra que as Coordenadas Cartesianas do Baricentro são: $\underline{\mathbf{G}(+7 ;+4) \text {. }}$

Figura 4 - Baricentro $(G)$ do triângulo escaleno de vértices A, B, D.

\subsection{Análise Gráfica do encontro das Mediatrizes ou Circuncentro (C)}

Conforme pode ser observado na Figura 5 a seguir, em qualquer triângulo, o Circuncentro (C) é o ponto de encontro das três mediatrizes (CARVALHO, 2005, p.25).. Mediatriz é o lugar geométrico dos pontos equidistantes aos extremos de um segmento de Reta. $\mathrm{CI}=$ Reta Mediatriz de AB; CJ = Reta Mediatriz de $\mathrm{AD}$ e $\mathrm{CK}=$ Reta Mediatriz de BD.
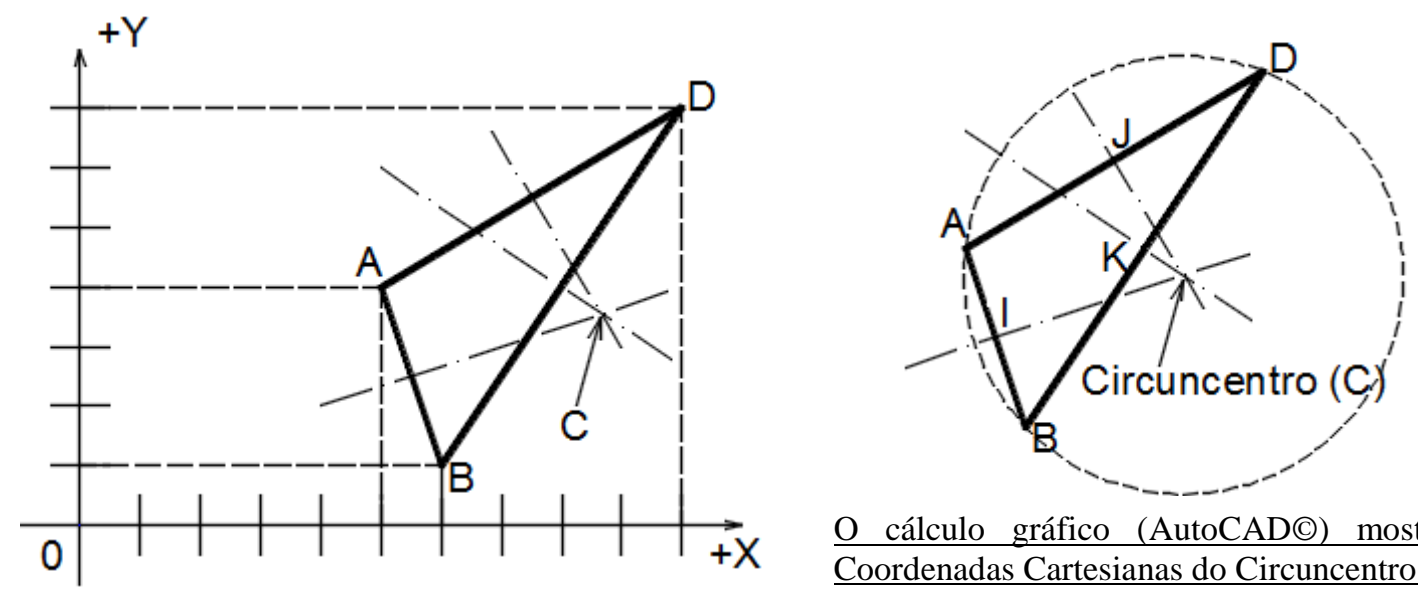

$\underline{\mathrm{O} \text { cálculo gráfico (AutoCADC) mostra que as }}$ Coordenadas Cartesianas do Circuncentro são: C (+8,666; + 3,555).

Figura 5 - Circuncentro $(C)$ do triângulo escaleno de vértices A, B, D. 


\subsection{Análise gráfica da Reta de Euler}

Uma vez definidas as Coordenadas Cartesianas dos pontos $\mathrm{O}, \mathrm{G}$ e C, faz-se uma representação gráfica do conjunto, usando-se o AutoCAD®, conforme apresentado na Figura 6 a seguir. O desenho confirma que: 1) existe uma Reta que passa pelos três pontos e que o ângulo que esta Reta faz com o eixo OX é $\beta=$ 14,949 , com o seu suplemento, ângulo $\alpha=165,051$. A tangente deste ângulo $\alpha(=-0,267)$ é o coeficiente angular "m”, que será analisado e calculado pelos conceitos da Geometria Analítica, descritos mais adiante, no parágrafo 5. 2) o Baricentro (G) está localizado entre o Ortocentro (O) e o Circuncentro (C). 3) a distância entre o Baricentro $(\mathrm{G})$ e o Ortocentro $(\mathrm{O})$ : $\mathrm{GO}=3,445$ é o dobro da distância entre o Baricentro $(\mathrm{G})$ e o Circuncentro: $\mathrm{GC}=1,7225$.

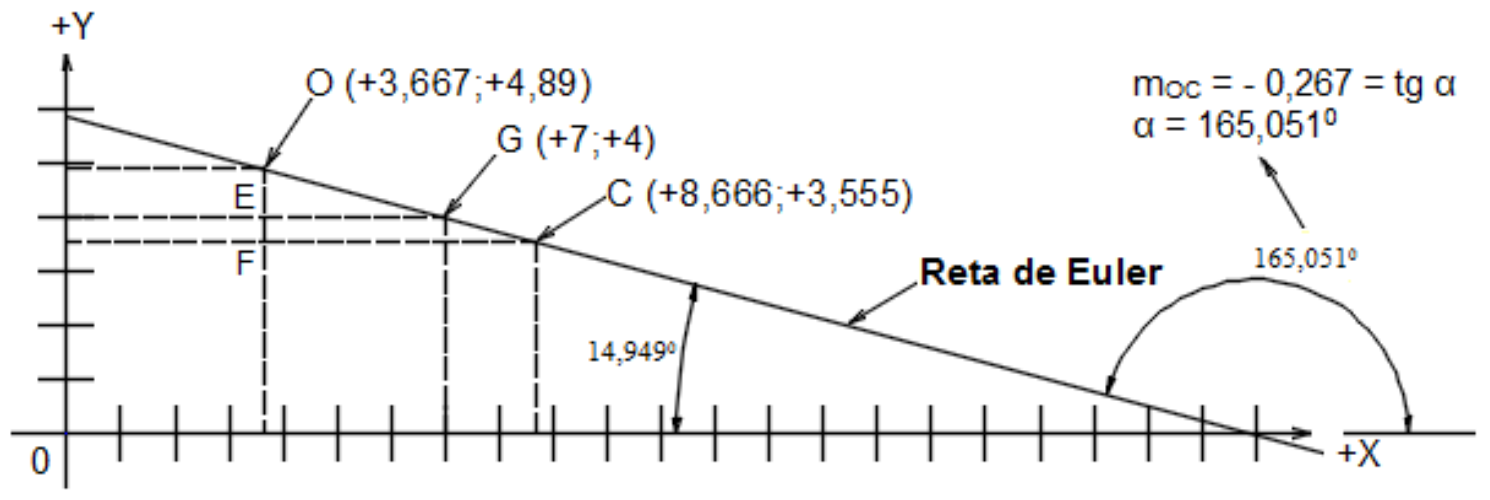

Figura 6 - Representação gráfica do conjunto, usando-se o AutoCAD®.

Esta é a Imagem Geométrica, com a comprovação da Reta de Euler, no triângulo considerado, executada pelo programa AutoCADC, bem como duas relações proporcionais, representadas na Figura 7 abaixo:

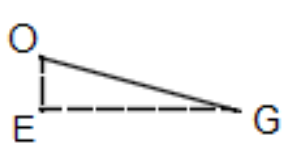

Semelhança entre os triângulos
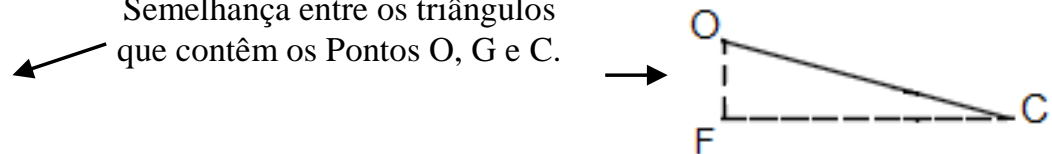

$\mathrm{OE}=0,89$ e $\mathrm{GE}=3,333: \mathrm{GE} / \mathrm{OE}=3,744$.

$\mathrm{OF}=1,335$ e $\mathrm{CF}=4,999: \mathrm{CF} / \mathrm{OF}=3,744$.

Figura 7 - Semelhança de triângulos $-\Delta \mathrm{OEG}$ e $\Delta \mathrm{OFC}$.

A análise da confirmação da semelhança dos triângulos OEG e OFC, comprova que os três Pontos $\mathrm{O}, \mathrm{G}$ e C, estão sobre a mesma Reta.

\section{A Reta de Euler analisada pelos conceitos da Geometria Analítica}

À luz da Geometria Analítica, a confirmação analítico-algébrica de que existe uma Reta que passa pelos três pontos notáveis, aqui estudados, exige as seguintes análises e cálculos: 1) Equação da Reta de cada um dos lados; 2) Determinação das Coordenadas Cartesianas do ponto médio de cada lado; 3) Equação da Reta de cada uma das Alturas; 4) Equação da Reta de cada uma das Medianas; 5) Equação da Reta de 
cada uma das Mediatrizes; 6) Determinação das Coordenadas Cartesianas do ponto de encontro das Alturas ou Ortocentro (ponto O); 7) Determinação das Coordenadas Cartesianas do ponto de encontro das Medianas ou Baricentro (ponto G); 8) Determinação das Coordenadas Cartesianas do ponto de encontro das Mediatrizes ou Circuncentro (ponto C) e 9) Determinação/confirmação de que a Reta de Euler passa pelos três pontos: $\mathrm{O}, \mathrm{G}$ e $\mathrm{C}$.

\subsection{Determinação da equação da Reta que passa por cada lado do triângulo}

Reta que passa pelo lado AB (Figura 8):

$\mathrm{A}(+5 ;+4), \mathrm{B}(+6 ;+1)$.

$\mathrm{m}_{\mathrm{AB}}=\left(\mathrm{y}_{\mathrm{A}}-\mathrm{y}_{\mathrm{B}}\right) /\left(\mathrm{x}_{\mathrm{A}}-\mathrm{x}_{\mathrm{B}}\right): \mathrm{m}_{\mathrm{AB}}=-3$.

$\mathrm{y}_{\mathrm{A}}-\mathrm{y}_{\mathrm{B}}=\mathrm{m}_{\mathrm{AB}}\left(\mathrm{x}_{\mathrm{A}}-\mathrm{x}_{\mathrm{B}}\right)$.

$\mathrm{y}-1=-3(\mathrm{x}-6): \mathrm{y}-1=-3 \mathrm{x}+18$ :

Reta $\mathrm{AB} \longrightarrow 3 \mathrm{x}+\mathrm{y}-19=0$

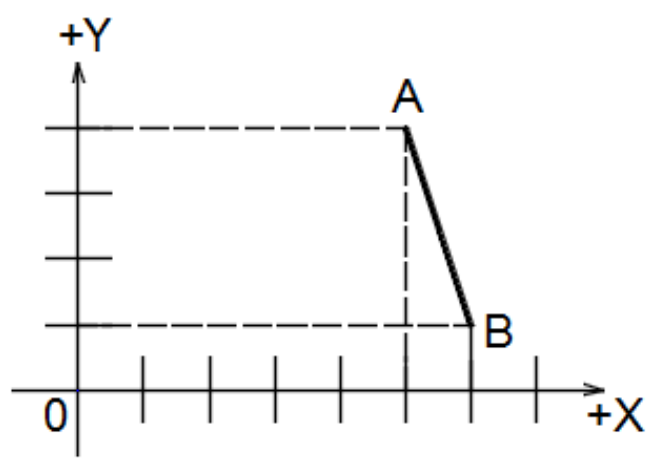

Figura 8 - Reta que passa pelo lado AB.

Reta que passa pelo lado AD (Figura 9):

$\mathrm{A}(+5 ;+4), \mathrm{D}(+10 ;+7)$.

$\mathrm{m}_{\mathrm{AD}}=\left(\mathrm{y}_{\mathrm{A}}-\mathrm{y}_{\mathrm{D}}\right) /\left(\mathrm{x}_{\mathrm{A}}-\mathrm{x}_{\mathrm{D}}\right): \mathrm{m}_{\mathrm{AD}}=+0,6$.

$\mathrm{y}_{\mathrm{A}}-\mathrm{y}_{\mathrm{D}}=\mathrm{m}_{\mathrm{AD}}\left(\mathrm{x}_{\mathrm{A}}-\mathrm{x}_{\mathrm{D}}\right)$.

$\mathrm{y}-7=+0,6(\mathrm{x}-10): \mathrm{y}-7=+0,6 \mathrm{x}-6$ :

Reta $\mathrm{AD} \longrightarrow 0,6 \mathrm{x}-\mathrm{y}+1=0$.

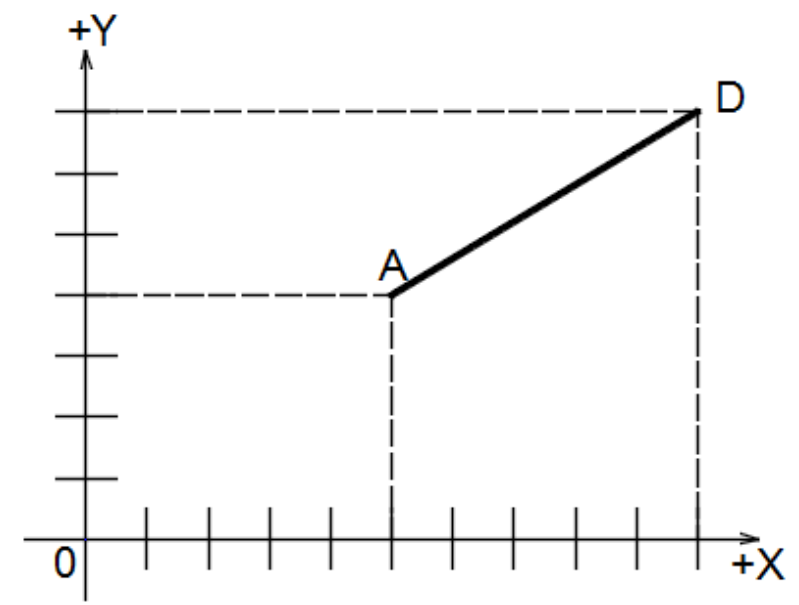

Figura 9 - Reta que passa pelo lado AD.

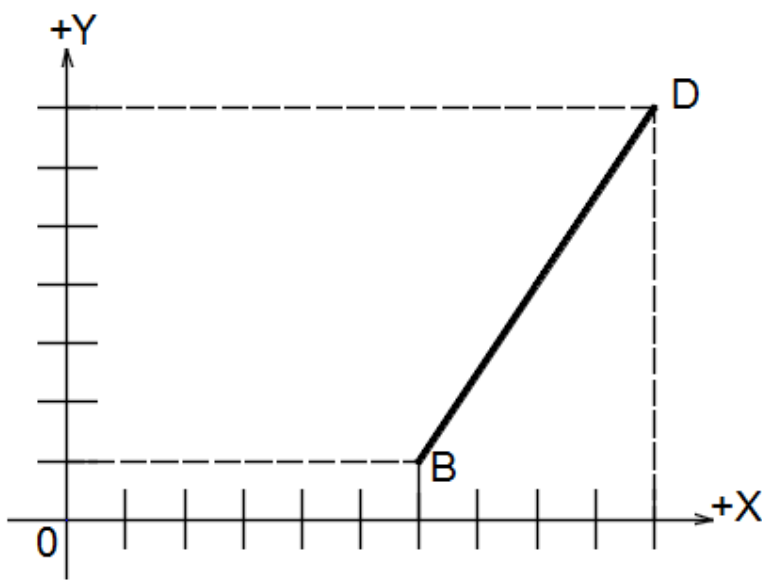

Reta que passa pelo lado BD (Figura 10):

$\mathrm{B}(+6 ;+1), \mathrm{D}(+10 ;+7)$.

$\mathrm{m}_{\mathrm{BD}}=\left(\mathrm{y}_{\mathrm{B}}-\mathrm{y}_{\mathrm{D}}\right) /\left(\mathrm{x}_{\mathrm{B}}-\mathrm{x}_{\mathrm{D}}\right): \mathrm{m}_{\mathrm{BD}}=+1,5$

$\mathrm{y}_{\mathrm{B}}-\mathrm{y}_{\mathrm{D}}=\mathrm{m}_{\mathrm{BD}}\left(\mathrm{x}_{\mathrm{B}}-\mathrm{x}_{\mathrm{D}}\right)$

$\mathrm{y}-7=+1,5(\mathrm{x}-10): \mathrm{y}-7=+1,5 \mathrm{x}-15$ :

Reta BD $\longrightarrow+1,5 \mathrm{x}-\mathrm{y}-8=0$.

Figura 10 - Reta que passa pelo lado BD. 


\subsection{Determinação das Coordenadas Cartesianas do ponto médio de cada lado}

\section{Ponto Médio do lado AB (ponto I):}

$$
\mathrm{I}=\left(\frac{\underline{x}_{A}+x_{B}}{2} ; \frac{y_{A}+y_{B}}{2}\right) ; I=(+5,5 ;+2,5)
$$

\section{Ponto Médio do lado AD (ponto J):}

$$
J=\left(\underline{x}_{\underline{A}}+x_{\underline{D}} ; \underline{y}_{\underline{A}}+y_{\underline{D}}\right) ; J=(+7,5 ;+5,5)
$$

\section{Ponto Médio do lado BD (ponto K):}

$$
\mathrm{K}=\left(\underline{\mathrm{x}}_{\underline{B}}+\mathrm{x}_{\underline{\mathrm{D}}} ; \underline{\underline{B}}_{\underline{B}}+\mathrm{y}_{\underline{\underline{D}}}\right) ; \mathrm{K}=(+8 ;+4)
$$

\subsection{Determinação da equação da Reta que passa por cada uma das Alturas}

Quando duas Retas são perpendiculares, o coeficiente angular de uma é igual ao oposto (sinal oposto) do inverso do outro coeficiente.

$\mathrm{DE}=$ Altura em relação a $\mathrm{AB}$, ou seja, a Reta que passa pelo lado $\mathrm{AB}(3 \mathrm{x}+\mathrm{y}-19=0)$ é perpendicular à Reta que passa pela Altura $\mathrm{DE}$. Como o coeficiente angular da Reta $\mathrm{AB}$ é $\mathrm{mAB}=-3$, o da Reta $\mathrm{DE}$ é: $\mathrm{mDE}=+1 / 3(+0,333)$, portanto a Reta que passa pela Altura $\mathrm{DE}$, tem coeficiente angular igual a $+0,333$ e passa pelo ponto $\mathrm{D}(+10 ;+7)$. Assim sendo tem-se:

$$
\mathrm{mDE}=(\mathrm{yD}-\mathrm{yE}) /(\mathrm{xD}-\mathrm{xE}):+0,333=(\mathrm{y}-7) /(\mathrm{x}-10) ; \text { portanto: }+\mathbf{0 , 3 3 3 x}-\mathbf{y}+\mathbf{3 , 6 6 7}=\mathbf{0} \text { é a }
$$
Equação da Reta que passa pela Altura DE.

$\mathrm{BH}=$ Altura em relação a $\mathrm{AD}$, ou seja, a Reta que passa pelo lado $\mathrm{AD}(-0,6 \mathrm{x}-\mathrm{y}+1=0)$ é perpendicular à Reta que passa pela Altura $\mathrm{BH}$. Como o coeficiente angular da Reta $\mathrm{AD}$ é $\mathrm{mAD}=+0,6$, o da Reta BH é: $\mathrm{mBH}=-1 / 0,6(-1,667)$, portanto a Reta que passa pela Altura BH, tem coeficiente angular igual a $-1,667$ e passa pelo ponto $\mathrm{B}(+6 ;+1)$. Assim sendo tem-se:

$\mathrm{mBH}=(\mathrm{yB}-\mathrm{yH}) /(\mathrm{xB}-\mathrm{xH}):-1,667=(\mathrm{y}-1) /(\mathrm{x}-6) ;$ portanto: $\mathbf{- 1 , 6 6 7 x}-\mathbf{y}+\mathbf{1 1}=\mathbf{0}$ é a Equação da Reta que passa pela Altura BH.

$\mathrm{AF}=$ Altura em relação a $\mathrm{BD}$, ou seja, a Reta que passa pelo lado $\mathrm{BD}(+1,5 \mathrm{x}-\mathrm{y}-8=0)$ é perpendicular à Reta que passa pela Altura $\mathrm{AF}$. Como o coeficiente angular da Reta $\mathrm{BD}$ é $\mathrm{mBD}=+1,5$, o da Reta AF é: $\mathrm{mAF}=-1 / 1,5(-0,667)$, portanto a Reta que passa pela Altura AF, tem coeficiente angular igual a $-0,667$ e passa pelo ponto A $(+5 ;+4)$. Assim sendo tem-se:

$\mathrm{mAF}=(\mathrm{yA}-\mathrm{yF}) /(\mathrm{xA}-\mathrm{xF}):-0,667=(\mathrm{y}-4) /(\mathrm{x}-5) ;$ portanto: $\mathbf{- 0 , 6 6 7} \mathbf{x}-\mathbf{y}+\mathbf{7 , 3 3 5}=\mathbf{0}$ é a Equação da Reta que passa pela Altura AF. 


\subsection{Determinação da equação da Reta de cada uma das Medianas}

Em verdade, cada Reta desta é a que passa por um vértice, cujas Coordenadas Cartesianas foram dadas, e pelo ponto médio do lado oposto ao vértice, sendo que este ponto médio foi calculado no parágrafo 5.2. Ou seja, trata-se de determinar a equação de uma Reta que passa por dois pontos, com as Coordenadas Cartesianas já conhecidas.

Determinação da equação da Reta Mediana DI, sendo D $(+10 ;+7)$ e I $(+5,5 ;+2,5)$ :

$\mathrm{m}_{\mathrm{DI}}=\left(\mathrm{y}_{\mathrm{D}}-\mathrm{y}_{\mathrm{I}}\right) /\left(\mathrm{x}_{\mathrm{D}}-\mathrm{x}_{\mathrm{I}}\right) ; \mathrm{m}_{\mathrm{DI}}=+1$.

$\mathrm{m}_{\mathrm{DI}}\left(\mathrm{x}_{\mathrm{D}}-\mathrm{x}_{\mathrm{I}}\right)=\left(\mathrm{y}_{\mathrm{D}}-\mathrm{y}_{\mathrm{I}}\right) ;+1\left(\mathrm{x}_{\mathrm{D}}-5,5\right)=\mathrm{y}_{\mathrm{D}}-2,5 ; \mathrm{x}_{\mathrm{D}}-5,5-\mathrm{y}_{\mathrm{D}}+2,5=0 ; \mathrm{x}-\mathrm{y}-3=0 ;$

Portanto: $+x-y-3=0$ é a Reta que passa pela Mediana DI.

Determinação da equação da Reta Mediana BJ, sendo B $(+6 ;+1)$ e J $(+7,5 ;+5,5)$ :

$\mathrm{m}_{\mathrm{BJ}}=\left(\mathrm{y}_{\mathrm{B}}-\mathrm{y}_{\mathrm{J}}\right) /\left(\mathrm{x}_{\mathrm{B}}-\mathrm{x}_{\mathrm{J}}\right) ; \mathrm{m}_{\mathrm{BJ}}=+3$.

$\mathrm{m}_{\mathrm{BJ}}\left(\mathrm{x}_{\mathrm{B}}-\mathrm{x}_{\mathrm{J}}\right)=\left(\mathrm{y}_{\mathrm{B}}-\mathrm{y}_{\mathrm{J}}\right) ;+3\left(\mathrm{x}_{\mathrm{B}}-7,5\right)=\mathrm{y}_{\mathrm{B}}-5,5 ;+3 \mathrm{x}_{\mathrm{B}}-22,5-\mathrm{y}_{\mathrm{B}}+5,5=0 ;+3 \mathrm{x}-\mathrm{y}-17=0$;

Portanto: $+3 \mathrm{x}-\mathrm{y}-17=0$ é a Reta que passa pela Mediana BJ.

Determinação da equação da Reta Mediana AK, sendo A $(+5 ;+4)$ e K $(+8 ;+4)$ :

$\mathrm{m}_{\mathrm{AK}}=\left(\mathrm{y}_{\mathrm{A}}-\mathrm{y}_{\mathrm{K}}\right) /\left(\mathrm{x}_{\mathrm{A}}-\mathrm{x}_{\mathrm{K}}\right) ; \mathrm{m}_{\mathrm{AK}}=0$.

Como m é o coeficiente angular da Reta, ou seja, a tangente do ângulo entre a Reta e o eixo OX, como m é igual a zero, significa que esta Reta AK é paralela ao eixo OX.

$\mathrm{m}_{\mathrm{AK}}\left(\mathrm{x}_{\mathrm{A}}-\mathrm{x}_{\mathrm{K}}\right)=\left(\mathrm{y}_{\mathrm{A}}-\mathrm{y}_{\mathrm{K}}\right)$;

$0=\mathrm{y}_{\mathrm{A}}-4$;

Ou seja; $y=+4$ é a Reta que passa pela Mediana AK.

\subsection{Determinação da equação da Reta de cada uma das Mediatrizes}

Cada Reta Mediatriz é perpendicular à Reta do referido lado e passa pelo seu ponto médio. Ou seja, cada uma destas equações envolve a determinação de uma Reta perpendicular a outra (Reta do lado) e passando por um determinado e conhecido ponto (ponto médio do lado). Quando duas Retas são perpendiculares, o coeficiente angular de uma é igual ao oposto (sinal oposto) do inverso do outro coeficiente.

Determinação da Reta CI, Mediatriz de AB, onde Reta $\mathrm{AB} \longrightarrow 3 \mathrm{x}+\mathrm{y}-19=0$, ponto médio I $(+5,5$; $+2,5)$, coeficiente angular $\mathrm{m}_{\mathrm{AB}}=-3$ e coeficiente angular $\mathrm{m}_{\mathrm{CI}}=-(-1 / 3)=+0,3333$.

$\mathrm{m}_{\mathrm{CI}}=+0,3333 ; \quad \mathrm{m}_{\mathrm{CI}}\left(\mathrm{x}-\mathrm{x}_{\mathrm{I}}\right)=\left(\mathrm{y}-\mathrm{y}_{\mathrm{I}}\right) ; \quad+0,3333(\mathrm{x}-5,5)=\mathrm{y}-2,5 ; \quad+0,3333 \mathrm{x}-1,8332-\mathrm{y}+2,5=$ 0 .

Ou seja: $+0,3333 x-y+0,6668=0$ é a Reta CI, Mediatriz de AB.

Determinação da Reta CJ, Mediatriz de AD, onde Reta $\mathrm{AD} \longrightarrow+0,6 \mathrm{x}-\mathrm{y}+1=0$, o ponto médio $\mathrm{J}$ $(+7,5 ;+5,5)$ e coeficiente angular $\mathrm{m}_{\mathrm{AD}}=+0,6$ e coeficiente angular $\mathrm{m}_{\mathrm{cj}}=-(1 /+0,6)=-1,6667$.

$\mathrm{m}_{\mathrm{cj}}=-1,6667 ; \quad \mathrm{m}_{\mathrm{CJ}}\left(\mathrm{x}-\mathrm{x}_{\mathrm{J}}\right)=\left(\mathrm{y}-\mathrm{y}_{\mathrm{J}}\right) ; \quad-1,6667(\mathrm{x}-7,5)=\mathrm{y}-5,5 ; \quad-1,6667 \mathrm{x}+12,5003-\mathrm{y}+5,5=$ 0 .

Ou seja: $+1,6667 x+y-18=0$ é a Reta CJ, Mediatriz de AD.

Determinação da Reta CK, Mediatriz de BD, onde Reta BD $\longrightarrow+1,5 \mathrm{x}-\mathrm{y}-8=0$, o ponto médio $\mathrm{K}$ $(+8 ;+4)$ e coeficiente angular $\mathrm{m}_{\mathrm{BD}}=+1,5$ e o coeficiente angular $\mathrm{m}_{\mathrm{CK}}=-(1 /+1,5)=-0,6667$.

$m_{\mathrm{CK}}=-0,6667 ; \quad \mathrm{m}_{\mathrm{CK}}\left(\mathrm{x}-\mathrm{x}_{\mathrm{K}}\right)=\left(\mathrm{y}-\mathrm{yK}_{\mathrm{K}}\right) ; \quad-0,6667(\mathrm{x}-8)=\mathrm{y}-4 ; \quad-0,667 \mathrm{x}+5,3336-\mathrm{y}+4=0$.

Ou seja: $+0,6667 x+y-9,3336=0$ é a Reta CK, Mediatriz de BD. 


\subsection{Determinação das Coordenadas Cartesianas do ponto de encontro das Alturas ou Ortocentro (O)}

O Ortocentro será determinado pela resolução de um sistema de equações. Como são três equações (três alturas) e duas incógnitas (x e y), serão resolvidos dois sistemas, com duas equações cada, achando-se os mesmos valores de x e y, em cada sistema: 1 ) Sistema com: equação da Reta que passa pela Altura DE (+ $0,3333 \mathrm{x}-\mathrm{y}+3,6666=0)$ e equação da Reta que passa pela Altura BH $(-1,6666 \mathrm{x}-\mathrm{y}+11=0) .2$ ) Sistema com: equação da Reta que passa pela Altura BH $(-1,667 x-y+11=0)$ e equação da Reta que passa pela Altura $\mathrm{AF}(-0,6666 \mathrm{x}-\mathrm{y}+7,335=0)$.

$\left\{\begin{array}{l}+0,3333 x-y+3,6666=0 ; \text { onde } y=+0,3333 x+3,6666 . \\ -1,667 x-y+11=0 ; \text { nesta equação, fazendo-se a substituição do y acima tem-se: }\end{array}\right.$

$-1,667 \mathrm{x}-0,333 \mathrm{x}-3,667+11=0 ;-2 \mathrm{x}-3,667+11=0 ;-2 \mathrm{x}=-7,333 ; \log 0: \mathrm{x}=+3,666$.

$\mathrm{y}=+0,333 \cdot 3,667+3,667 ; \log : \mathrm{y}=+4,89$.

$$
\left\{\begin{array}{l}
-1,667 x-y+11=0 ; \text { onde } y=-1,667 x+11 \\
-0,667 x-y+7,335=0 ; \text { nesta equação, fazendo-se a substituição do y acima tem-se: }
\end{array}\right.
$$

$-0,667 \mathrm{x}+1,667 \mathrm{x}-11+7,335=0 ;+\mathrm{x}-3,665=0 ; \log 0 ; \mathrm{x}=+3,666$.

$\mathrm{y}=-1,667.3,665+11 ; \operatorname{logo}: \mathrm{y}=+4,89$

As Coordenadas Cartesianas do Ortocentro $(\mathrm{O})$ são: $\mathbf{O}(\mathbf{+ 3 , 6 6 7} ; \mathbf{+} \mathbf{4 , 8 9})$, ou seja, os mesmos valores encontrados pela análise gráfica feita no AutoCAD.

\subsection{Determinação das Coordenadas Cartesianas do ponto de encontro das Medianas ou Baricentro (G)}

Apesar de ter-se três equações, em uma delas, a Reta $\mathrm{AK}$, a equação é y $=+4$, ou seja, basta apenas resolver um sistema com as equações das outras duas medianas: DI e BJ, para achar o valor de $\mathrm{x}$ e confirmar o de y, que é igual a +4 .

$$
\begin{aligned}
& \left\{\begin{array}{l}
+\mathrm{x}-\mathrm{y}-3=0 ; \mathrm{x}=\mathrm{y}+3 . \\
+3 \mathrm{x}-\mathrm{y}-17=0
\end{array}\right. \\
& +3(\mathrm{y}+3)-\mathrm{y}-17=0 ; \quad 3 \mathrm{y}+9-\mathrm{y}-17=0 ; \quad+2 \mathrm{y}=+8 ; \quad \mathrm{y}=+4 \mathrm{e} \mathrm{x}=+7 .
\end{aligned}
$$

As Coordenadas Cartesianas do Baricentro (ponto G) são: $\mathbf{G}(+\mathbf{7} ; \mathbf{+ 4}$ ), ou seja, os mesmos valores encontrados pela análise gráfica feita no AutoCAD.

\subsection{Determinação das Coordenadas Cartesianas do ponto de encontro das Mediatrizes ou Circuncentro (C)}

O Circuncentro será determinado pela resolução de um sistema de equaçães. Como são três equações (três Mediatrizes) e duas incógnitas (x e y), serão resolvidos dois sistemas, com duas equações cada, achando-se os mesmos valores de x e y, em cada sistema: 1) Sistema com: equação da Reta que passa pela Mediatriz CI $(+0,3333 \mathrm{x}-\mathrm{y}+0,6668=0)$ e equação da Reta que passa pela Mediatriz CJ $(+$ $1,6667 \mathrm{x}+\mathrm{y}-18=0)$. 2) Sistema com: equação da Reta que passa pela Mediatriz CI $(+0,3333 \mathrm{x}-\mathrm{y}+$ $0,6668=0)$ e equação da Reta que passa pela Mediatriz CK $(+0,6667 x+y-9,3336=0)$.

$$
\begin{aligned}
& \left\{\begin{array}{l}
+0,3333 \mathrm{x}-\mathrm{y}+0,6668=0 ; \mathrm{y}=+0,333 \mathrm{x}+0,6668 . \\
+1,6667 \mathrm{x}+\mathrm{y}-18=0
\end{array}\right. \\
& \left\{\begin{array}{l}
+1,6667 \mathrm{x}+\mathrm{y}-18=0 ; \mathrm{y}=-1,6667 \mathrm{x}+18 . \\
+0,6667 \mathrm{x}+\mathrm{y}-9,3336=0 .
\end{array} \quad \longrightarrow \mathrm{x}=+8,666 \mathrm{e} \mathrm{y}=+3,555\right.
\end{aligned}
$$

As coordenadas Cartesianas do Circuncentro são: $\mathbf{C}(+\mathbf{8 , 6 6 6} ;+\mathbf{3 , 5 5 5})$, ou seja, os mesmos valores encontrados pela análise gráfica feita no AutoCAD. 


\subsection{Determinação/confirmação de que a Reta de Euler passa pelos pontos: O, G e C}

Como já foram determinadas as Coordenadas Cartesianas dos três pontos, a confirmação de que existe uma Reta (de Euler) que passa por estes pontos poderá ser feita, de duas formas: 1) determinando-se a equação de uma Reta que passa pelos pontos O (Ortocentro) e G (Baricentro) e a equação de uma Reta que passa pelos pontos $\mathrm{O}$ (Ortocentro) e C (Circuncentro), segundo o coeficiente angular. Como os três pontos pertencem à mesma Reta (de Euler), as duas equações terão o mesmo coeficiente angular; 2) Outra forma de confirmar que três pontos são colineares, ou seja, que estão sobre a mesma Reta, é através do Determinante de Sarrus. Neste parágrafo serão feitas as duas análises.

\section{A Reta de Euler, segundo sua equação definida pelo coeficiente angular m}

\subsection{Reta entre $O(+3,667 ;+4,89)$ e $G(+7 ;+4)$ :}

$\mathrm{m}_{\mathrm{OG}}=\left(\mathrm{y}_{\mathrm{O}}-\mathrm{y}_{\mathrm{G}}\right) /\left(\mathrm{x}_{\mathrm{O}}-\mathrm{x}_{\mathrm{G}}\right) ; \mathrm{m}_{\mathrm{OG}}=(+4,89-4) /(+3,667-7) ; \mathrm{m}_{\mathrm{OG}}=-0,267$.

$\mathrm{y}-\mathrm{y}_{\mathrm{G}}=\mathrm{m}_{\mathrm{OG}}\left(\mathrm{x}-\mathrm{x}_{\mathrm{G}}\right) ; \mathrm{y}-4=-0,267(\mathrm{x}-7) ; \mathrm{y}-4=-0,267 \mathrm{x}+1,869$ :

$+0,267 \mathrm{x}+\mathrm{y}-5,869=0 \longleftarrow$ Equação da Reta OG.

\subsection{Reta entre $O(+3,667 ;+4,89)$ e $C(+8,666 ;+3,555)$ :}

$m_{O C}=\left(y_{O}-y_{C}\right) /\left(x_{O}-x_{C}\right) ; \quad m_{O C}=(+4,89-3,555) /(+3,667-8,666) ; m_{O C}=-0,267$.

$y-y_{C}=m_{O C}\left(x-x_{C}\right) ; y-3,555=-0,267(x-8,666) ; \quad y-3,555=-0,267 x+2,314:$

$+0,267 \mathrm{x}+\mathrm{y}-5,869=0 \longleftarrow$ Equação da Reta OC.

Como, tanto os coeficientes angulares, quanto as equações são iguais, concluí-se que os três pontos: $\mathrm{O}(+3,667 ;+4,89), \mathrm{G}(+7 ;+4)$ e $\mathrm{C}(+8,666 ;+3,555)$, pertencem à mesma Reta, ou seja:

A equação da Reta de Euler, do triângulo citado, é: $+0,267 x+y-5,869=0$.

Além de todas as características analisadas analiticamente, esta Reta de Euler, apresenta dois outros pontos típicos e com propriedades específicas, conforme Figura 11 a seguir: o primeiro é o ponto onde a Reta corta o eixo dos Y, ou seja, onde a abscissa x é igual a zero. Neste ponto tem-se o valor do coeficiente linear desta Reta, que é igual a $+5,869$. O segundo ponto é onde a Reta corta o eixo dos X, ou seja, onde a ordenada y é igual a zero. Neste ponto tem-se $\mathrm{x}=+21,981$.

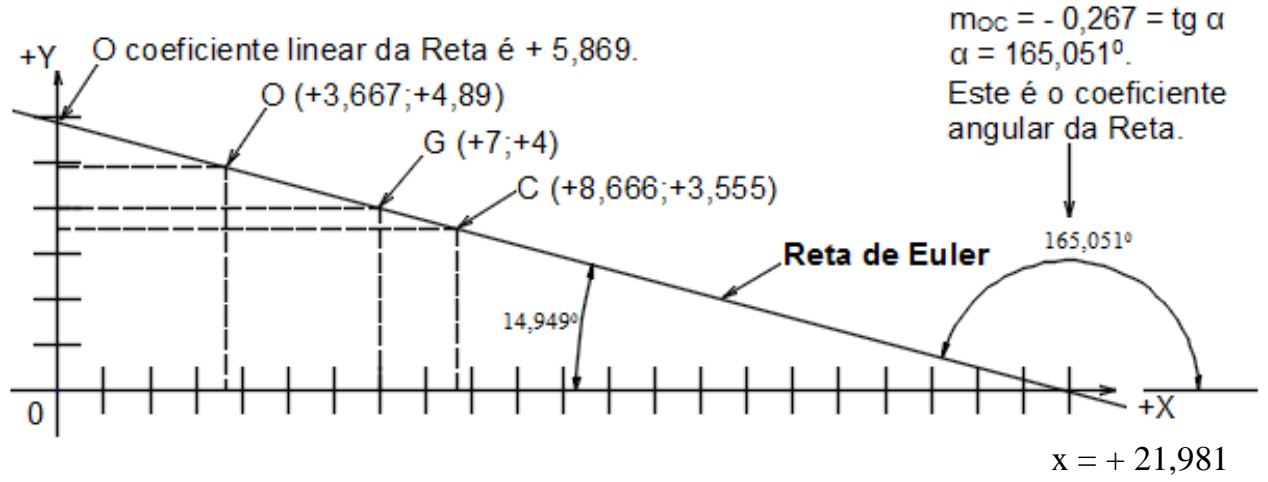

Figura 11 - Imagem Geométrica da Reta de Euler no triângulo considerado. 


\section{Confirmação da equação da Reta de Euler, pela Regra do Determinante $(\Delta)$ de Sarrus:}

Segundo esta Regra, se o Determinante $(\Delta)$ composto pelas Coordenadas Cartesianas dos três pontos, como adiante descrito, for igual a zero, os três pontos estão alinhados, ou seja, pertencem à mesma Reta.

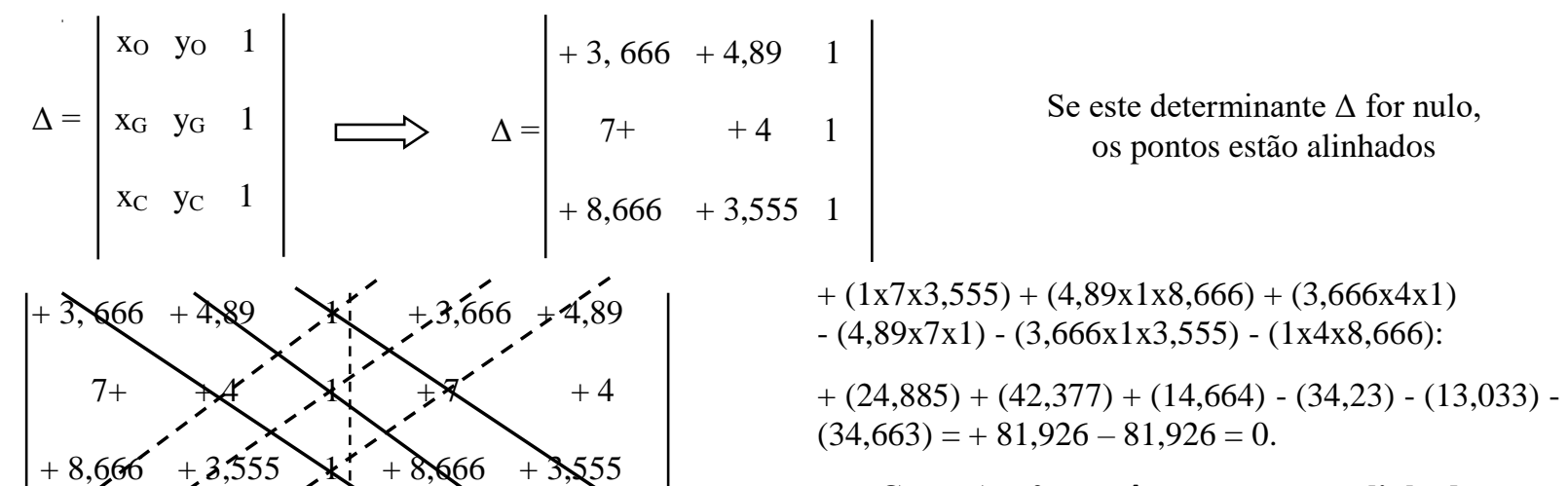

Como $\Delta=0$, os três pontos estão alinhados.

\section{Considerações Finais}

Considerando as análises gráfica e analítica, baseadas nas Referências a seguir, pode-se confirmar e concluir que: 1) em triângulos escalenos e isósceles existe uma Reta (de Euler) que passa pelos seguintes pontos notáveis: Ortocentro $(\mathrm{O})$, Baricentro $(\mathrm{G})$ e Circuncentro (C). Esta Reta é chamada de Reta de Euler. 2) Na Reta de Euler, o Baricentro $(G)$ está localizado entre o Ortocentro (O) e o Circuncentro (C). 3) A distância entre o Baricentro $(\mathrm{G})$ e o Ortocentro $(\mathrm{O})$ é o dobro da distância entre o Baricentro $(\mathrm{G})$ e o Circuncentro (C). 4) Existe uma Circunferência com centro no encontro das Mediatrizes (o Circuncentro) e que passa pelos três vértices de um triângulo.

\section{Referências}

ABRANTES, José. A Geometria Analítica aplicada nos espaços $\mathrm{R}_{2}$ e $\mathrm{R}_{3}$. Desenvolvendo o Raciocínio Lógico-Matemático, a Visão Espacial e as Habilidades Gráficas ou Pictóricas. Rio de Janeiro: Independente, 2017. ISBN: 978-85-903210-5-7.

AUTODESK, INC. AutoCAD 2015 - Software.

CARVALHO, Benjamin de Araújo. 3.ed. 32 reimp. Desenho Geométrico. Rio de Janeiro: Imperial Novo Milênio, 2005. 